\title{
Intraoperative Frozen Cytology of Central Nervous System Neoplasms: An Ancillary Tool for Frozen Diagnosis
}

Myunghee Kang* · Dong Hae Chung* Na Rae Kim · Hyun Yee Cho Seung Yeon $\mathrm{Ha} \cdot$ Sangho Lee Jungsuk An · Jae Yeon Seok Gie-Taek Yie ${ }^{1}$. Chan Jong Yoo ${ }^{1}$ Sang Gu Lee ${ }^{1}$. Eun Young Kim ${ }^{1}$ Woo Kyung $\mathrm{Kim}^{1}$ · Seong Son ${ }^{1}$ Sun Jin Sym² ${ }^{2}$ Dong Bok Shin ${ }^{2}$ Hee Young Hwang ${ }^{3}$. Eung Yeop $\mathrm{Kim}^{3}$ Kyu Chan Lee

Departments of Pathology and ${ }^{1}$ Neurosurgery, 2Division of Medical Oncology, Department of Internal Medicine, Departments of ${ }^{3}$ Radiology and ${ }^{4}$ Radiation Oncology, Gil Medical Center, Gachon University College of Medicine, Incheon, Korea

Received: October 1, 2018

Revised: November 7, 2018

Accepted: November 10, 2018

Corresponding Author

Na Rae Kim, MD

Department of Pathology, Gil Medical Center,

Gachon University College of Medicine,

21 Namdong-daero 774 beon-gil, Namdong-gu,

Incheon 21565, Korea

Tel: +82-32-460-3073

Fax: +82-32-460-2394

E-mail: clara_nrk@gilhospital.com

*Myunghee Kang and Dong Hae Chung contributed equally to this work.
Background: Pathologic diagnosis of central nervous system (CNS) neoplasms is made by comparing light microscopic, immunohistochemical, and molecular cytogenetic findings with clinicoradiologic observations. Intraoperative frozen cytology smears can improve the diagnostic accuracy for CNS neoplasms. Here, we evaluate the diagnostic value of cytology in frozen diagnoses of CNS neoplasms. Methods: Cases were selected from patients undergoing both frozen cytology and frozen sections. Diagnostic accuracy was evaluated. Results: Four hundred and fifty-four cases were included in this retrospective single-center review study covering a span of 10 years. Five discrepant cases (1.1\%) were found after excluding 53 deferred cases ( 31 cases of tentative diagnosis, 22 cases of inadequate frozen sampling). A total of 346 cases of complete concordance and 50 cases of partial concordance were classified as not discordant cases in the present study. Diagnostic accuracy of intraoperative frozen diagnosis was $87.2 \%$, and the accuracy was 98.8\% after excluding deferred cases. Discrepancies between frozen and permanent diagnoses $(n=5,1.1 \%)$ were found in cases of nonrepresentative sampling $(n=2)$ and misinterpretation $(n=3)$. High concordance was observed more frequently in meningeal tumors $(97 / 98,99 \%)$, metastatic brain tumors (51/52, 98.1\%), pituitary adenomas (86/89, 96.6\%), schwannomas (45/47, 95.8\%), high-grade astrocytic tumors (47/58, 81\%), low grade astrocytic tumors (10/13, 76.9\%), non-neoplastic lesions $(23 / 36,63.9 \%)$, in decreasing frequency. Conclusions: Using intraoperative cytology and frozen sections of CNS tumors is a highly accurate diagnostic ancillary method, providing subtyping of CNS neoplasms, especially in frequently encountered entities.

Key Words: Crush cytology; Central nervous system; Neoplasm; Frozen sections
Intraoperative diagnosis of central nervous system (CNS) neoplasms is limited by marked artifacts due to ice crystals during freezing and the difficulty of observing nuclear details of the tumor cells. ${ }^{1}$ Since the first study of intraoperative cytological smears of CNS lesions in 1930 by Eisenhardt and Cushing, ${ }^{2}$ the use of smear cytology has increased in the diagnosis of CNS neoplasms. Recently, diagnosing CNS neoplasms has shown a rapid transition to molecular incorporation for layered diagnoses. However, cytology continues to provide important clues in frozen diagnoses of CNS neoplasms. ${ }^{3}$ Few studies exist relating to cytomorpho- logic significance, especially on frozen diagnoses, and inadequate experience and resultant poor interpretations hinder conclusive diagnoses.

Herein, we evaluate the diagnostic accuracy of intraoperative combined cytology and frozen sections in the diagnosis of CNS neoplasms.

\section{MATERIALS AND METHODS}

We retrieved 454 cases that underwent both intraoperative 
frozen cytologic smears and frozen section slides of brain and cord from the archives of the Department of Pathology at a tertiary referral hospital from 2006 to 2015. In cases where only small specimens were submitted, only frozen cytology was examined. The frozen cytology was prepared by squash or touch smears, which were immediately immersed in $95 \%$ ethanol for two minutes and stained with hematoxylin and eosin (H\&E). The remaining specimens were frozen sectioned; the tissue was processed in a cryostat at $-15^{\circ} \mathrm{C}$. Thin sections $(5-\mu \mathrm{m}$ thickness $)$ were made and then stained using H\&E. ${ }^{4}$ The frozen diagnoses were compared to permanent diagnoses. Disease entities were classified according to the 2007 World Health Organization (WHO) classification of CNS tumors with the combined application of new concepts from the 2016 revised edition, e.g., solitary fibrous tumors/hemangiopericytoma. ${ }^{5,6}$ To determine discordance, we categorized cases as complete concordance, partial concordance, discordance, and deferred cases including inadequate samples. Cases were considered complete concordance when specified frozen entities were the same as final ones, and as partial concordance when entities were not conclusive, but suggestive upon frozen diagnosis. Cases were considered deferred when, for example, glioblastoma was reported as suspicious for glioblastoma. We arbitrarily defined major and minor entities when the incidence was more than $10 \%$ or less than $10 \%$, respectively. Comparison between diagnostic accuracy was assessed using chisquare tests in SPSS ver. 17 (SPSS Inc., Chicago, IL, USA), and p < .05 was considered significant. All procedures were in accordance with the Helsinki Declaration of 1964 and later versions. This study is approved by Gil Medical Center's Institutional Review Board (GBIRB2016-323), and informed consent from patients was waived.

\section{RESULTS}

\section{Diseases diagnosed through smear cytological examination}

The most commonly encountered diseases were meningiomas, followed by (in order of decreasing frequency) pituitary adenomas, low- and high-grade astrocytic gliomas, and metastatic tumors. The cytologic features are shown in Fig. 1. The results are shown in Table 1.

\section{Concordance rate by disease entities}

The accuracy of cytology was $87.2 \%$ (396/454), and reached 98.8\% (396/401) after excluding deferred cases $(\mathrm{n}=53)$. Five cases were categorized as discordant (Table 2, Fig. 2). Minor disease entities showed low complete concordance (less than 50\%), compared with major disease entities showing high concordance (range, $69 \%$ to $95.5 \%$ ). When excluding metastatic brain tumors, disease entities comprising more than $10 \%$ of the total showed statistically significant diagnostic accuracy by frozen cytology, compared to less common tumors (meningioma including atypical ones, pituitary adenoma, high-grade glioma and schwannoma $)(\mathrm{p}<.001)$.

\section{Deferred cases}

Tentative diagnoses in the present study were $6.8 \%(n=31)$ and were categorized as deferred diagnoses, except in the case of inadequate samples. These results are shown in Table 3. The rates of deferred cases between major disease entities (i.e., more than $10 \%$ of incidence) and non-major disease entities were significantly different $(\mathrm{p}<.001)$.

\section{DISCUSSION}

The present study retrospectively assessed the use of both cytology and frozen sections, so direct comparison of the frozen cytology and frozen sections is limited. The use of intraoperative frozen sections of CNS neoplasms has long been an important diagnostic tool, but agreement varies among histopathological entities and shows low sensitivity in diagnosing low-grade tumors and edematous lesions, compared to intraoperative frozen cytology. Various limitations in frozen diagnoses of CNS neoplasms exist in both frozen cytologic smears and frozen sections. Due to their soft, friable, and watery nature, frozen sections frequently have marked freezing artifacts and ice crystals, resulting in obscured nuclear details. These limitations may be resolved by frozen cytology, except for CNS lesions with a firm consistency such as schwannomas, metastatic carcinomas, or non-neoplastic inflammatory lesions. In the latter lesions, only frozen sections can maintain architectural morphology and subsequently yield high diagnostic accuracy. Here, we retrospectively assessed diagnostic utility using both frozen cytology and frozen sections; discrepancies between frozen diagnosis and final diagnosis may be ascribed to sampling error and incorrect evaluation of histologic typing or grading. Except for grading, previous studies showed the most sensitive yield was achieved in tumors including astrocytoma, meningioma, oligodendroglioma, metastatic tumor, glioblastoma, and ependymoma in descending order. ${ }^{3,8}$ Glial tumors show heterogeneous distribution; ${ }^{9}$ high grade, i.e., grades III and IV astrocytic tumors may be diagnosed as low-grade tumors in frozen diagnosis. ${ }^{10}$ These errors can be avoided by taking samples from multiple portions of the tumors. ${ }^{11}$ In frozen cytology and sections 

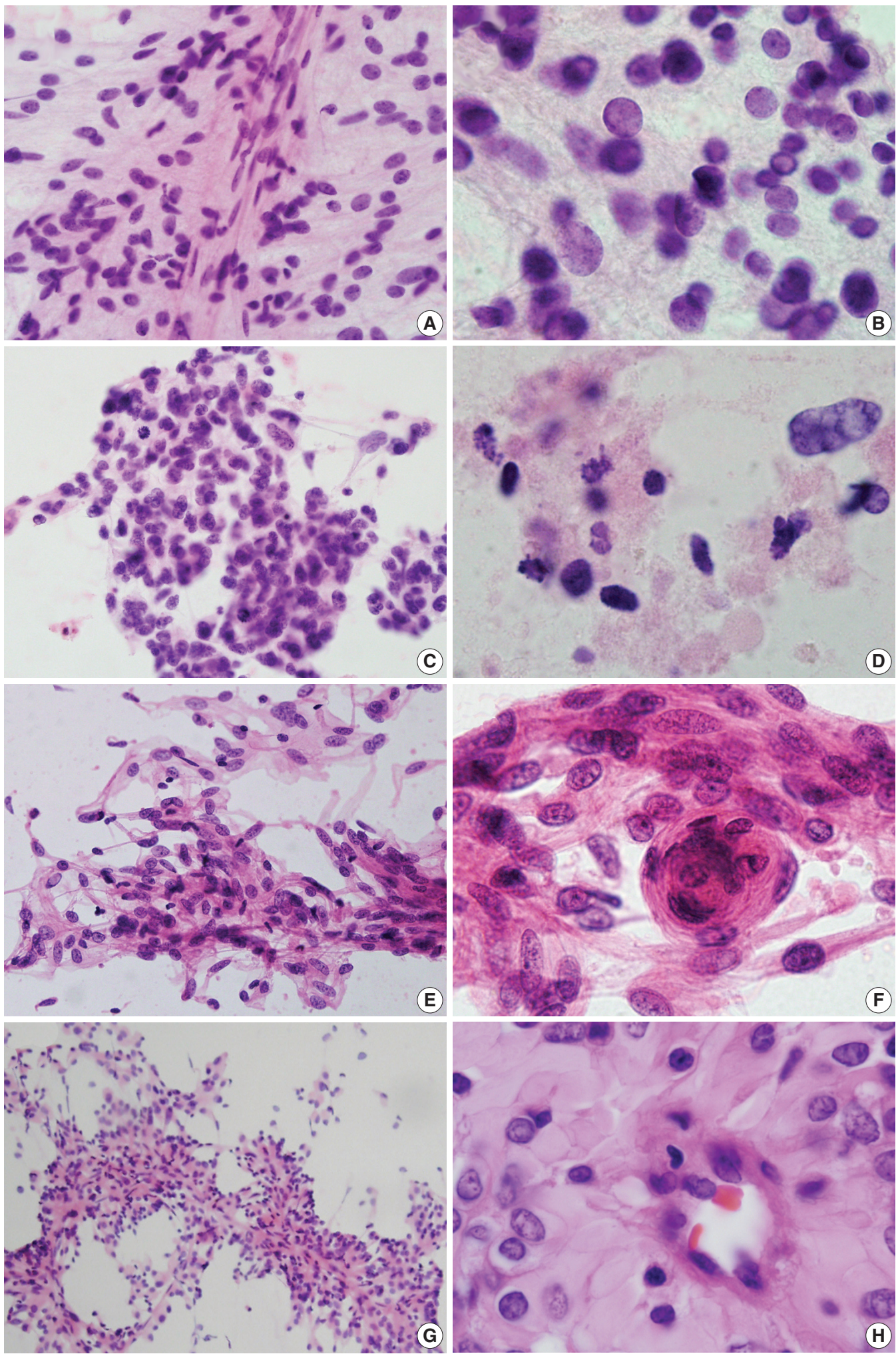

Fig. 1. (A, B) Ependymoma. Spindle-shaped tumor cells around thin capillaries. Note fibrillary cytoplasm and occasional nuclear inclusion. (C, D) Glioblastoma. Clusters show ovoid to slightly elongated hyperchromatic nuclei with occasional mitosis. Note necrosis in the extracellular area. $(E, F)$ Fibrous meningioma. Ovoid to spindle cells have round to elongated nuclei with fine chromatin. Note nuclear pseudoinclusion and meningothelial whorls. $(G, H)$ Rhabdoid and papillary meningioma. Papillae have thick collagenous cytoplasm rather than fibrillary features that are commonly found in ependymoma. 
Table 1. Diagnostic comparison between frozen cytological diagnoses and permanent histologic diagnoses

\begin{tabular}{|c|c|c|c|c|c|}
\hline Disease entities & $\begin{array}{c}\text { Completely } \\
\text { concordant } \\
\text { cases }\end{array}$ & $\begin{array}{l}\text { Partially } \\
\text { concordant } \\
\text { cases }\end{array}$ & $\begin{array}{l}\text { Sum } \\
\text { of concordant } \\
\text { cases }\end{array}$ & $\begin{array}{l}\text { Discordant } \\
\text { cases }\end{array}$ & $\begin{array}{c}\text { Deferred cases } \\
\text { including inadequate } \\
\text { samples }\end{array}$ \\
\hline Meningioma $(n=98)$ & 91 (92.9) & $6(6.1)$ & $97(99.0)$ & $1(1)$ & 0 \\
\hline Pituitary adenoma $(n=89)$ & $85(95.5)$ & $1(1.1)$ & $86(96.6)$ & 0 & $3(3.4)$ \\
\hline \multicolumn{6}{|l|}{ Glial tumor } \\
\hline Astrocytic tumor, high grade $(n=58)$ & $40(69.0)$ & $7(12.1)$ & $47(81.1)$ & $2(3.4)$ & $9(15.5)$ \\
\hline Astrocytic tumor, low grade $(n=13)$ & $4(30.8)$ & $6(46.2)$ & $10(76.9)$ & $1(7.7)$ & $2(15.4)$ \\
\hline Ependymoma $(n=10)$ & $3(30.0)$ & $1(10.0)$ & $4(40.0)$ & 0 & $6(60.0)$ \\
\hline Oligodendroglioma $(n=3)$ & $2(66.7)$ & $1(33.3)$ & $3(100)$ & 0 & 0 \\
\hline Anaplastic oligodendroglioma $(n=3)$ & $3(100)$ & 0 & $3(100)$ & 0 & 0 \\
\hline \multicolumn{6}{|l|}{ Neurogenic tumor } \\
\hline Schwannoma $(n=47)$ & 38 (80.9) & $7(14.9)$ & $45(95.7)$ & 0 & $2(4.3)$ \\
\hline Neurofibroma $(n=2)$ & 0 & $2(100)$ & $2(100)$ & 0 & 0 \\
\hline Dysembryoplastic neuroepithelial tumor $(n=2)$ & $1(50)$ & $1(50)$ & $2(100)$ & 0 & 0 \\
\hline Germ cell tumor $(n=3)$ & $1(33.3)$ & 0 & $1(33.3)$ & $1(33.3)$ & $1(33.3)$ \\
\hline Malignant SFT/HP $(n=3)$ & $1(33.3)$ & 0 & $1(33.3)$ & 0 & $2(66.7)$ \\
\hline Hematologic malignancy $(n=9)$ & $3(33.3)$ & $5(55.6)$ & $8(88.9)$ & 0 & $1(11.1)$ \\
\hline Metastatic tumor $(n=52)$ & $46(88.5)$ & $5(9.6)$ & $51(98.1)$ & 0 & $1(1.9)$ \\
\hline Other malignant tumors $(n=6)$ & $1(16.7)$ & $2(33.3)$ & $3(50)$ & 0 & $3(50)$ \\
\hline Other benign tumors $(n=20)$ & $8(40.0)$ & $2(10.0)$ & $10(50)$ & 0 & $10(50.0)$ \\
\hline Non-neoplastic lesions $(n=36)$ & $19(52.8)$ & $4(11.1)$ & $23(63.9)$ & 0 & $13(36.1)$ \\
\hline Total cases $(n=454)$ & $346(76.2)$ & $50(11.0)$ & $396(87.2)$ & $5(1.1)$ & $53(11.7)$ \\
\hline
\end{tabular}

Values are presented as number (\%).

SFT/HP, solitary fibrous tumor/hemangiopericytoma.

Table 2. Comparison between frozen and permanent diagnoses and analysis of reason for diagnostic error of discordant cases

\begin{tabular}{llll}
\hline $\begin{array}{l}\text { Case } \\
\text { No. }\end{array}$ & \multicolumn{1}{c}{ Frozen cytology and section diagnosis } & \multicolumn{1}{c}{ Permanent diagnosis } & Reason for error \\
\hline 1 & Atypical glial cell proliferation with necrotic debris, favor reactive change & Diffuse astrocytoma & Misinterpretation \\
2 & Consistent with benign glial tumor & Glioblastoma & Misinterpretation \\
3 & Histiocytic lesion & Glioblastoma & Nonrepresentative sample \\
4 & Ependymoma & Papillary and rhabdoid meningioma & Misinterpretation \\
5 & Squamous epithelium, suggestive of craniopharyngioma & Immature teratoma & Nonrepresentative sample \\
\hline
\end{tabular}

of glial lesions, discrimination between normal brain reactive gliosis versus low-grade gliomas (WHO grades I and II) is difficult; evaluation of true cellularity is limited in both frozen cytology and frozen sections. Increased gliosis is insufficient to diagnose glioma. Features showing unevenly distributed astrocytes with mild nuclear irregularities, no discernible cytoplasm, high nuclear to cytoplasmic ratios, and hyperchromatic chromatin favor lowgrade glioma. Low cellular smears composed of spindle cells, especially the peripheral areas of high-grade gliomas, are difficult to interpret. Radiation injury may mimic glioblastoma by cytological findings such as bizarre nuclear features. ${ }^{12}$ Necrosis and vascular endothelial proliferation are reliable cytologic findings of high-grade glioma. ${ }^{10,13,14}$ Because they share clinicoradiologic findings, glioblastoma should be distinguished from metastasis and lymphoma. Glioblastoma is characterized by highly malignant cells and endovascular proliferation, i.e., a bulbous collection of overlapped and haphazardly oriented nuclei attached to vessels on frozen cytology. ${ }^{15}$ On the other hand, lymphoma cytology lacks endovascular proliferation, shows no tumor cells within the walls of blood vessels, exhibits a spread of atypical, round nuclei with vesicular, open chromatin and distinct nucleoli, and displays abundant lymphoglandular bodies with tingible body macrophages in the background. Pleomorphic xanthoastrocytoma belongs to astrocytic tumors showing marked nuclear pleomorphism with no mitotic activity in frozen cytology. ${ }^{16}$

Among CNS neoplasms showing spindle cell morphology, frozen cytology samples of meningothelial neoplasms are notorious for having diverse cytologic findings. Most conventional, lowgrade meningiomas show nuclear inclusion or vesicular nuclear clearing and thick collagenous cytoplasm as well as syncytial whorls. ${ }^{17}$ Compared to low-grade meningiomas, high-grade meningiomas such as rhabdoid or papillary types (WHO grade III) 

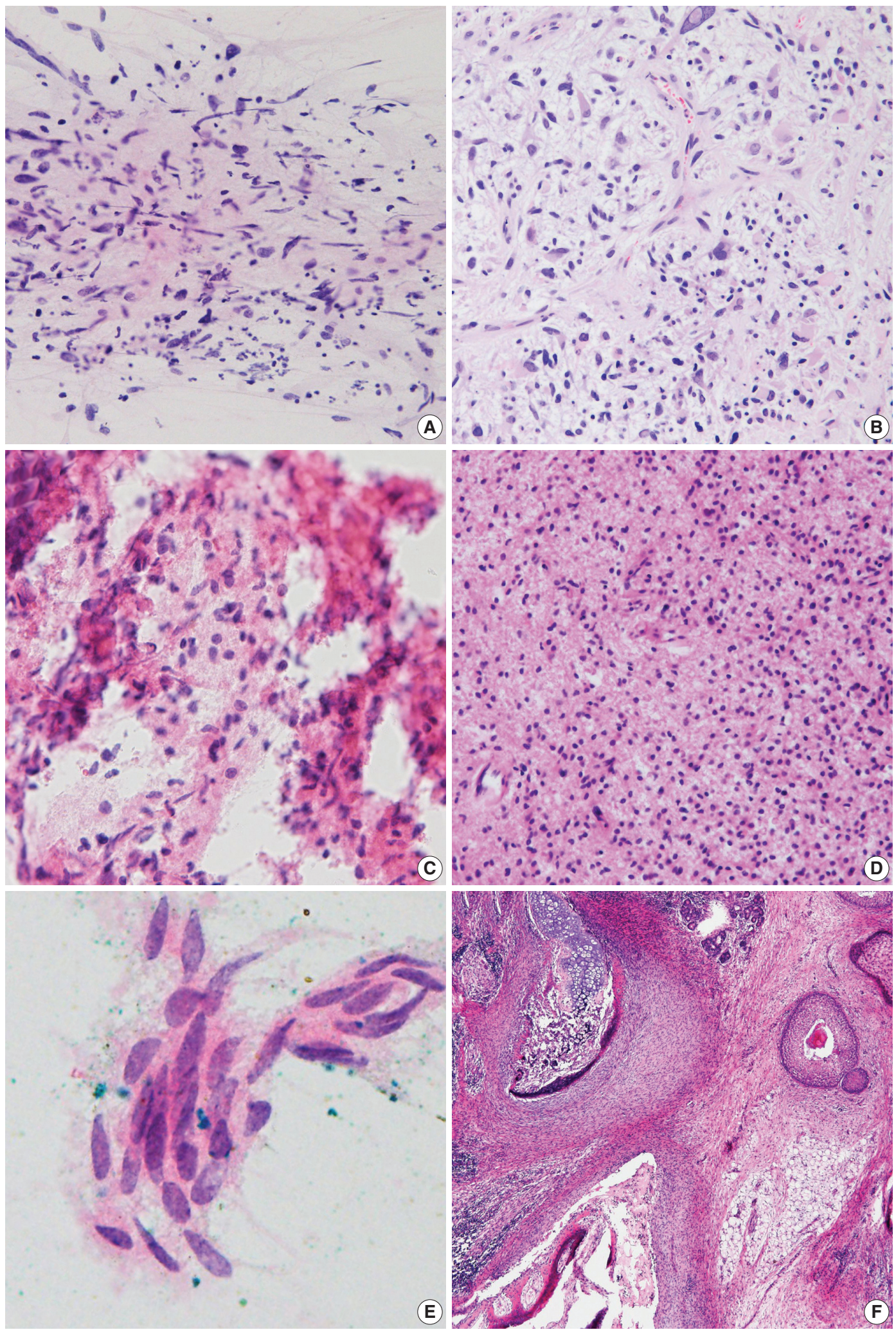

Fig. 2. Discordant cases. (A, B) Spindle cells with occasional atypical cells are mixed with karyorrhectic debris (A), and glioblastoma is diagnosed in the permanent section (B). (C, D) A tiny focus of round cells shows eosinophilic fibrillary cytoplasm in a bloody background (C), and diffuse astrocytoma is diagnosed in the permanent section (D). (E) Only one cluster of squamoid epithelium is seen in frozen smears. (F) Immature teratoma with neuroepithelium is finally diagnosed in permanent section. 
Table 3. Deferred cases excluding inadequate specimens in the frozen diagnoses

\begin{tabular}{|c|c|}
\hline Disease entities & No. \\
\hline Pituitary adenoma & 3 \\
\hline Astrocytic tumor, low grade & 2 \\
\hline Gliomatosis cerebri & 1 \\
\hline Pilomyxoid astrocytoma & 1 \\
\hline Astrocytic tumor, high grade & 8 \\
\hline Glioblastoma & 5 \\
\hline Anaplastic astrocytoma & 3 \\
\hline Ependymoma & 6 \\
\hline Ependymoma & 3 \\
\hline Cellular ependymoma & 1 \\
\hline Myxopapillary ependymoma & 2 \\
\hline Schwannoma & 1 \\
\hline Germ cell tumor & 1 \\
\hline Mature teratoma & 1 \\
\hline Malignant solitary fibrous tumor/hemangiopericytoma & 2 \\
\hline Hematologic malignancy & 1 \\
\hline Plasmacytoma & 1 \\
\hline Metastatic tumor & 1 \\
\hline Metastatic adenocarcinoma & 1 \\
\hline Other malignant tumor & 2 \\
\hline Hemangioblastoma & 2 \\
\hline Other benign tumors & 4 \\
\hline Vascular malformation & 1 \\
\hline Hemangioma & 2 \\
\hline Rathke's cleft cyst & 1 \\
\hline Non-neoplastic lesions & 1 \\
\hline No diagnostic abnormality & 1 \\
\hline
\end{tabular}

may share cytological findings of ependymoma because meningiomas of rhabdoid or papillary types show few meningothelial whorls and predominant perivascular rosette-like arrangements that were misdiagnosed as ependymoma. ${ }^{18}$ Nuclear inclusions on cytologic smears may be shared by these two disease entities. ${ }^{15,18}$ Eosinophilic hyalinized cytoplasm and hyaline globules of rhabdoid meningioma should be distinguished from atypical teratoid/ rhabdoid tumors, germinoma, or embryonal carcinomas. ${ }^{19}$ Even anaplastic meningioma shows pleomorphic cells, mimicking carcinoma or sarcoma. Schwannomas lack whorls and have elongated, wavy cytoplasm. ${ }^{15,20}$ Distinguishing meningioma and schwannoma is not difficult in most cases. However, uncommon locations such as intracerebral or sellar schwannoma require careful intraoperative diagnoses. ${ }^{21}$

Round cell lesions such as oligodendroglioma containing abundant thin capillary structures may easily exclude astrocytic lesions due to a perinuclear halo artifact that is typically seen in permanent formalin-fixed processing of oligodendrogliomas. ${ }^{14,22}$ Cytological smears of oligodendroglioma-mimicking round cell tumors such as central neurocytoma, hemangioblastoma, metastatic clear cell renal cell carcinoma, and dysembryoplastic neuroepithelial tumor should be differentiated from oligodendroglioma and small cell glioblastoma. ${ }^{23,24}$ Central neurocytoma, an oligodendroglioma-mimicker, is a well-differentiated tumor showing features of neuronal differentiation that may mimic the frozen histology of oligodendroglioma, clear cell ependymoma, or even necrotic glioblastoma due to eosinophilic acellular fibrillary areas masquerading as necrotic portions under frozen histologic sections, despite the small round cells having fine, granular nuclear chromatin and cytoplasm. ${ }^{20,25}$ When pathologists observe cytological findings such as arborizing thin-walled vasculature, calcification, or mucoid foci, these shared features should be distinguished from clear cell ependymoma, central neurocytoma, or oligodendroglioma. Nuclear features that include a salt-pepper appearance are features of neurocytoma, but cellular areas with alternating acellular fibrillary neuropil-rich areas may mimic or be easily misinterpreted as ependymal rosettes. ${ }^{24}$ Nuclear details of ependymomas are distinguished from those of neurocytomas by the presence of nuclear grooves and nuclear inclusions. ${ }^{25}$ On observing nuclear features, round to oval nuclei with stippled chromatin, as well as nuclear grooves and intracytoplasmic lumina, are seen in ependymomas. ${ }^{18}$ Clear cell ependymoma, one of the oligodendroglioma-mimickers, may pose a significant diagnostic challenge because cytological findings of a few cells with a clear cytoplasm and eccentric nucleus are unconventional for ependymoma; ${ }^{20}$ conventional type ependymomas show a distinct cytomorphology, but less-epithelial variants of ependymomas, such as tanycytic variants, may resemble astrocytic tumors even in cytological smears. ${ }^{26}$ Ependymomas having acellular perivascular fibrillary pseudorosettes are composed of round-shaped, monotonous, cohesive tumor cells. ${ }^{25}$ However, cytological findings such as loosely arranged monotonous round-shaped tumor cells with small nuclei and stippled chromatin have provided diagnostic distinguishing points in cases with acellular fibrillary areas scattered in the cytology smears. ${ }^{1}$ The WHO classification of CNS neoplasms classifies ependymomas into three grades, considering parameters of cellularity, pleomorphism, mitosis, necrosis, and microvascular proliferation. ${ }^{6}$ Cellular pleomorphism and microvascular proliferation were the most reliable indicators of WHO grade III ependymomas compared to mitosis or necrosis patterns. ${ }^{27}$ Intraoperative cytological characteristics of ependymomas are well described, but cytologic characteristics by grading are rarely documented.

Intraoperative frozen sections provide an important tool, but agreement varies among histopathological entities and is lower in low-grade tumors than in high-grade tumors, compared to intraoperative cytology. ${ }^{28}$ For example, frozen diagnosis of menin- 
giomas is not different from intraoperative cytology. Because the present study retrospectively assessed the use of both cytology and frozen sections, direct comparison of the two is limited. The cytologic characteristics described here with frozen sections revealed that diagnostic accuracy of intraoperative frozen diagnosis was 87.2\% (396/454), and the accuracy was elevated up to $98.8 \%$ after excluding deferred cases. Two out of five discordant cases were ascribed to sampling error taken from nonrepresentative tissue portions. The remaining three cases were misinterpreted as reactive gliosis or low-grade tumors in bloody backgrounds. Bloody backgrounds may contribute to false negative diagnoses, so clinical confirmation is always desirable in unusual smears. With respect to correct grading of glial tumors, concordance of glial tumors was low. Distinguishing between reactive gliosis and lowgrade gliomas is limited and difficult, by both frozen cytology and frozen section. ${ }^{3}$ Non-neoplastic CNS lesions mimicking glial tumors include demyelinating diseases and tissue-destructive lesions such as infarctions, abscesses, radio-necrosis, and areas with resolving hemorrhage. ${ }^{22,29}$

These cytomorphologic features in conjunction with the clinical history and radiologic findings provided an accurate diagnosis in $87.2 \%$ of the cases in the present study, similar to previous studies reporting a diagnostic accuracy varying from $86 \%$ to $97.3 \%$. $^{1,30}$ Relatively high concordance rates of major disease entities in the present study are related with frequent exposure and experience with those CNS neoplasms. This diagnostic accuracy can be improved through knowledge of clinical and neuroimaging correlation as well as experienced pathologists.

Given the clinical significance of intraoperative tentative diagnoses of CNS lesions, it is important to examine cytological smears and frozen sections combined with clinicoradiologic findings to increase diagnostic accuracy. Intraoperative frozen diagnoses using combined cytology and frozen sections encompass limitations in layered diagnosis according to revised upcoming WHO classifications and inevitable deferral of grading, and awareness of these pitfalls is important for neuropathologists and neurosurgeons. Despite the limitations of retrospective studies, this study describes the 10-year retrospective experience of prompt and accurate intraoperative frozen section diagnoses in CNS lesions with the combined use of smear cytology and highlights its diagnostic value as a reliable method.

\section{ORCID}

Myunghee Kang: https://orcid.org/0000-0003-4083-888X

Dong Hae Chung: https://orcid.org/0000-0002-4538-0989
Na Rae Kim: https://orcid.org/0000-0003-2793-6856 Hyun Yee Cho: https://orcid.org/0000-0003-3603-5750 Seung Yeon Ha: https://orcid.org/0000-0001-7071-1623 Sangho Lee: https://orcid.org/0000-0003-4636-7521 Jungsuk An: https://orcid.org/0000-0003-0312-2460 Jae Yeon Seok: https://orcid.org/0000-0002-9567-6796 Gie-Taek Yie: https://orcid.org/0000-0002-8706-7253 Chan Jong Yoo: https://orcid.org/0000-0002-2680-7692 Sang Gu Lee: https://orcid.org/0000-0001-9943-4906 Eun Young Kim: https://orcid.org/0000-0002-4942-8425 Woo Kyung Kim: https://orcid.org/0000-0002-0974-903X Seong Son: https://orcid.org/0000-0002-2815-9908 Sun Jin Sym: https://orcid.org/0000-0002-1340-6291 Dong Bok Shin: https://orcid.org/0000-0002-6872-4043 Hee Young Hwang: https://orcid.org/0000-0002-1152-5684 Eung Yeop Kim: https://orcid.org/0000-0002-9579-4098 Kyu Chan Lee: https:/orcid.org/0000-0002-6295-3996

\section{Author Contributions}

Conceptualization: NRK.

Data curation: KCL.

Formal analysis: MK, DHC.

Investigation: SJS, DBS.

Project administration: NRK.

Resources: SGL, EYK (Eun Young Kim), CJY, GTY, WKK, SS. Supervision: NRK, HYC, SYH.

Validation: JA, SL, JYS.

Visualization: SGL, EYK (Eung Yeop Kim), HYH.

Writing —original draft: NRK, MK, DHC.

Writing—review \& editing: NRK, MK, DHC.

\section{Conflicts of Interest}

The authors declare that they have no potential conflicts of interest.

\section{REFERENCES}

1. Marshall LF, Adams H, Doyle D, Graham DI. The histological accuracy of the smear technique for neurosurgical biopsies. J Neurosurg 1973; 39: 82-8.

2. Eisenhardt L, Cushing H. Diagnosis of intracranial tumors by supravital technique. Am J Pathol 1930; 6: 541-52.

3. Gal AA, Cagle PT. The 100-year anniversary of the description of the frozen section procedure. JAMA 2005; 294: 3135-7.

4. Cahill EM, Hidvegi DF. Crush preparations of lesions of the central nervous system: a useful adjunct to the frozen section. Acta Cytol 
1985; 29: 279-85.

5. Louis DN, Ohgaki H, Wiestler OD, Cavenee WK. WHO classification of tumours of the central nervous system. 4th ed. Lyon: IARC Press, 2016.

6. Louis DN, Ohgaki H, Wiestler OD, Cavenee WK. WHO classification of tumours of the central nervous system. Lyon: IARC Press, 2007.

7. Folkerth RD. Smears and frozen sections in the intraoperative diagnosis of central nervous system lesions. Neurosurg Clin N Am 1994; 5: 1-18.

8. Rushing EJ, Wesseling P. Towards an integrated morphological and molecular WHO diagnosis of central nervous system tumors: a paradigm shift. Curr Opin Neurol 2015; 28: 628-32.

9. Inagawa $\mathrm{H}$, Ishizawa $\mathrm{K}$, Hirose $\mathrm{T}$. Qualitative and quantitative analysis of cytologic assessment of astrocytoma, oligodendroglioma and oligoastrocytoma. Acta Cytol 2007; 51: 900-6.

10. Kang M, Kim NR, Chung DH, Yoo CJ. Crush cytologic findings of a cerebral granular cell astrocytoma. Acta Cytol 2012; 56: 571-5.

11. Shioyama T, Muragaki Y, Maruyama T, Komori T, Iseki H. Intraoperative flow cytometry analysis of glioma tissue for rapid determination of tumor presence and its histopathological grade: clinical article. J Neurosurg 2013; 118: 1232-8.

12. Lampert PW, Davis RL. Delayed effects of radiation on the human central nervous system: "early" and "late" delayed reactions. Neurology 1964; 14: 912-7.

13. Lee S, Kim NR, Chung DH, Yee GT, Cho HY. Squash cytology of a dural-based high-grade chondrosarcoma may mimic that of glioblastoma in the central nervous system. Acta Cytol 2015; 59: 219-24.

14. Plesec TP, Prayson RA. Frozen section discrepancy in the evaluation of central nervous system tumors. Arch Pathol Lab Med 2007; 131: $1532-40$.

15. Lee HS, Tihan T. The basics of intraoperative diagnosis in neuropathology. Surg Pathol Clin 2015; 8: 27-47.

16. Yamada SM, Murakami H, Tomita Y, et al. Glioblastoma multiforme versus pleomorphic xanthoastrocytoma with anaplastic features in the pathological diagnosis: a case report. Diagn Pathol 2016; 11: 65 .

17. Seok JY, Kim NR, Cho HY, Chung DH, Yee GT, Kim EY. Crush cytology of microcystic meningioma with extensive sclerosis. Korean J
Pathol 2014; 48: 77-80.

18. Jeong J, Kim NR, Lee SG. Crush cytology of a primary intraspinal rhabdoid papillary meningioma: a case report. Acta Cytol 2013; 57: 528-33.

19. Raisanen J, Hatanpaa KJ, Mickey BE, White CL 3rd. Atypical teratoid/rhabdoid tumor: cytology and differential diagnosis in adults. Diagn Cytopathol 2004; 31: 60-3.

20. Kobayashi S. Meningioma, neurilemmoma and astrocytoma specimens obtained with the squash method for cytodiagnosis: a cytologic and immunochemical study. Acta Cytol 1993; 37: 913-22.

21. Kim NR, Suh YL. Primary intrasellar schwannoma: a case report. Korean J Pathol 2002; 36: 274-7.

22. Plesec TP, Prayson RA. Frozen section discrepancy in the evaluation of nonneoplastic central nervous system samples. Ann Diagn Pathol 2009; 13: 359-66.

23. Can B, Akpolat I, Meydan D, Üner A, Kandemir B, Söylemezoğlu F. Fine-needle aspiration cytology of metastatic oligodendroglioma: case report and literature review. Acta Cytol 2012; 56: 97-103.

24. Hernández-Martínez SJ, De Leíja-Portilla JO, Medellín-Sánchez R. Cytologic features during intraoperative assessment of central neurocytoma: a report of three cases and review of the literature. Acta Cytol 2013; 57: 400-5.

25. Deb P, Manu V, Pradeep H, Bhatoe HS. Intraparenchymal clear cell ependymoma. J Cytol 2011; 28: 73-6.

26. Chaudhuri PM, Chaudhuri S, Chakrabarty D, et al. Squash cytology of tanycytic ependymoma: a report of two cases. Diagn Cytopathol 2017; 45: 270-3.

27. Wang M, He X, Chang Y, Sun G, Thabane L. A sensitivity and specificity comparison of fine needle aspiration cytology and core needle biopsy in evaluation of suspicious breast lesions: a systematic review and meta-analysis. Breast 2017; 31: 157-66.

28. Mahe E, Ara S, Bishara M, et al. Intraoperative pathology consultation: error, cause and impact. Can J Surg 2013; 56: E13-8.

29. Monabati A, Kumar PV, Kamkarpour A. Intraoperative cytodiagnosis of metastatic brain tumors confused clinically with brain abscess: a report of three cases. Acta Cytol 2000; 44: 437-41.

30. Chand P, Amit S, Gupta R, Agarwal A. Errors, limitations, and pitfalls in the diagnosis of central and peripheral nervous system lesions in intraoperative cytology and frozen sections. J Cytol 2016; 33: 93-7. 